\title{
Method to Improve Azo-Compound (AAPH)-Induced Hemolysis of Erythrocytes for Assessing Antioxidant Activity of Lipophilic Compounds
}

\author{
Yusuke Nuruki, ${ }^{\dagger}$ Haruka Matsumoto, ${ }^{\ddagger}$ Miho Tsukada, ${ }^{\S}$ Haruka Tsukahara,${ }^{\$}$ Tokuko Takajo, \\ Kazunori Tsuchida, and Kazunori Anzai* \\ Division of Physical and Analytical Chemistry, Faculty of Pharmaceutical Sciences, Nihon Pharmaceutical \\ University; Saitama 362-0806, Japan. \\ Received July 14, 2020; accepted October 8, 2020
}

We examined the method of oxidative hemolysis for assessment of antioxidant activity of various compounds, especially lipophilic compounds. 2,2'-Azobis(amidinopropane) dihydrochloride (AAPH) was used as the source of free radicals for the oxidative hemolysis of horse erythrocytes. We found that absorbance at $540 \mathrm{~nm}$ is not appropriate for monitoring AAPH-induced hemolysis. Instead, we should use absorbance at $523 \mathrm{~nm}$ (an isosbestic point), because AAPH oxidizes the oxygenated hemoglobin to methemoglobin and absorbance at $540 \mathrm{~nm}$ does not correctly reflect the amount of released hemoglobin by AAPH-induced hemolysis. The corrected method of AAPH-induced hemolysis was applicable to assess the antioxidant activity of various hydrophilic compounds such as ascorbic acid, (-)-epicatechin, and edaravone. For the assessment of antioxidant activity of lipophilic compounds, we need appropriate dispersing agents for these lipophilic compounds. Among several agents tested, 1,2-dimiristoyl-sn-glycero-3-phosphocholine (DMPC) liposome at a concentration of $0.34 \mathrm{mM}$ was found to be useful. Exogenous $\alpha$-tocopherol incorporated using DMPC liposome as a dispersing agent was shown to protect erythrocytes from AAPH-induced hemolysis in a concentration-dependent manner.

Key words antioxidant; hemolysis; erythrocyte; $2,2^{\prime}$-azobis(amidinopropane) dihydrochloride (AAPH)

\section{Introduction}

Reactive oxygen species (ROS) attack various components of the living body, such as proteins, nucleic acids, and lipids, on which various harmful effects are exerted.1) To prevent the harmful effects of ROS, organisms have antioxidant enzymes in the body, such as superoxide dismutase (SOD) and peroxidase, as well as various antioxidative components such as $\alpha$-tocopherol ( $\alpha$-Toc) and uric acid. In addition, various antioxidative compounds in foods, beverages, and nutritional supplements may be useful for preventing or reducing the harmful effects of ROS. A variety of techniques have been proposed to measure antioxidant activity of complex compound mixtures including ESR spin trapping technique, ${ }^{2,3)}$ oxygen radical absorbance capacity (ORAC) assay, ${ }^{4,5}$ trolox equivalent antioxidant capacity (TEAC) assay, ${ }^{6}$ and ferric reducing antioxidant power (FRAP) assay. ${ }^{7)}$

Lipid peroxidation of biomembranes is an initial step of oxidative cell damage. Therefore, it is possible to utilize lipid peroxidation as an indicator of oxidative stress. If we measure lipid peroxidation and related phenomena with and without a testing compound, we may evaluate the an-

\footnotetext{
${ }^{\dagger}$ Present address: Kawaguchi Kogyo General Hospital; 1-18-15 Aoki, Kawaguchi, Saitama 332-0031, Japan.

† Present address: Matsumotokiyoshi Co. Ltd.; 9-1 Shinmatsudohigashi, Matsudo, Chiba 270-8501, Japan.

${ }^{\S}$ Present address: Shichihongi Pharmacy; 1904-1 Shichihongi, Kamisatomachi, Kodama-gun, Saitama 369-0306, Japan.

${ }^{\$}$ Present address: Chuoh Pharmacy; 11-21 Minamitakasago-cho, Utsunomiya, Tochigi 321-0143, Japan.
}

tioxidant activity of the testing compound. Previous studies reported that hemolysis of erythrocytes was induced by 2,2'-azobis(amidinopropane) dihydrochloride (AAPH), a water-soluble azo compound, when added to a suspension of erythrocytes and that this hemolysis was suppressed by antioxidants. ${ }^{8-11)}$ Oxidation of erythrocytes serves as a model for the oxidation damage of biological membranes and the free radicals generated in an aqueous phase induce chain oxidation of lipids and proteins in erythrocyte membranes that cause hemolysis. ${ }^{7,9,10)}$ AAPH is an oxidation agent, and thermal decomposition of an AAPH molecule generates two carbon-centered alkyl radical molecules, which then react with oxygen to produce peroxyl radical molecules ${ }^{8,9,12)}$ and alkoxyl radical molecules ${ }^{13)}$ with oxidation activity. Many studies have used AAPH-induced oxidative hemolysis to measure antioxidant activity of various compounds, especially complex mixture compounds. ${ }^{14-30)}$ In the present study, we re-examined the methodology of AAPH-induced oxidative hemolysis and propose an improved method for evaluating antioxidant activity of various compounds, especially lipophilic compounds, using AAPH-induced hemolysis of erythrocytes.

Results

When the erythrocyte suspension was mixed with the AAPH solution, absorbance at about 540 and $570 \mathrm{~nm}$ of the erythrocyte supernatant after centrifugation increased with time. The spectral pattern with absorption bands at these wavelengths corresponded to the spectrum of oxygenated hemoglobin $\left(\mathrm{HbO}_{2}\right),{ }^{31-33)}$ indicating that hemolysis was induced by the reaction with AAPH. In addition to the increase in the 
A)

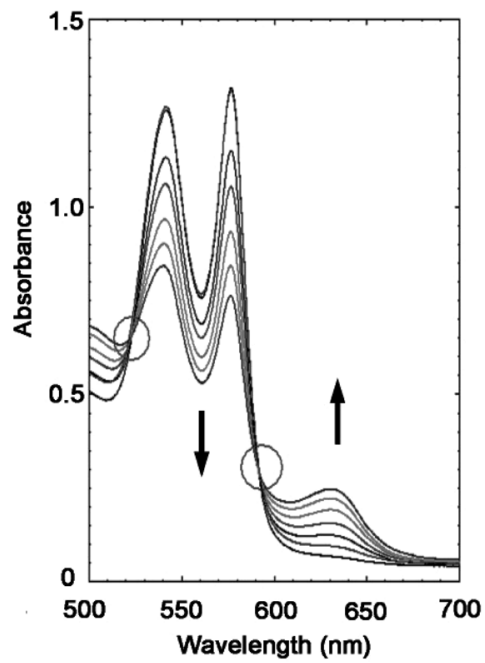

B)

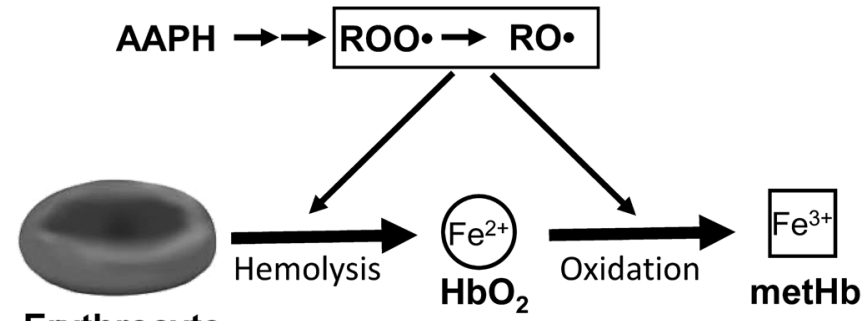

Erythrocyte

Fig. 1. Effects of AAPH on Erythrocytes

A) Hemoglobin was released from horse blood by hemolysis of erythrocytes with water, and then AAPH was added. Time course absorption spectra were measured at $0,15,30,45,60,75$, and $90 \mathrm{~min}$ after mixing with AAPH. The absorbance around $550 \mathrm{~nm}$ decreased with time, whereas absorbance around $630 \mathrm{~nm}$ increased. Two isosbestic points shown in circles were observed at 523 and $591 \mathrm{~nm}$. B) A conceptual diagram of the effects of AAPH on erythrocytes. An AAPH molecule is thermally decomposed to two molecules of 2-methyl-amidinopropane radicals which are converted to peroxyl radicals (ROO) by the reaction with a molecular oxygen and alkoxyl radicals (RO') by the decomposition of ROO*. ROO' and RO induce hemolysis by oxidative membrane damage of erythrocytes as the first step. $\mathrm{ROO}^{\circ}$ and $\mathrm{RO}^{\circ}$ also oxidize the released hemoglobin $\left(\mathrm{HbO}_{2}\right)$ with $\mathrm{Fe}^{2+}$ in the heme group to methemoglobin (metHb) with $\mathrm{Fe}^{3+}$ heme as the second step.

two peaks, we found that a new absorption band appeared at $630 \mathrm{~nm}$ after $90 \mathrm{~min}$ of the reaction. When the spectrum of the sample obtained from the complete hemolysis of erythrocytes induced by the addition of water in the presence of AAPH was compared with that in the absence of AAPH, we found that the intensity of the two peaks of the AAPH-treated erythrocytes were lower than that of the untreated erythrocytes. This observation suggested that a new species was produced by the reaction of $\mathrm{HbO}_{2}$ with AAPH. To examine this further, we first prepared a sample of complete hemolysis by treating erythrocytes with water, and then AAPH was added. As shown in Fig. 1A, the intensity of the bands at $541 \mathrm{~nm}$ and $576 \mathrm{~nm}$ decreased with time, while the intensity of the newly observed band at $630 \mathrm{~nm}$ increased. The reaction of AAPH was not very fast. Indeed, a small change was observed in the absorbance at 541 and $576 \mathrm{~nm}$ even at $1 \mathrm{~d}$ after mixing, indicating that the reaction did not reach equilibrium (data not shown). Two isosbestic points were observed at 523 and $591 \mathrm{~nm}$. If we measure the absorbance at 541 or $576 \mathrm{~nm}$, the

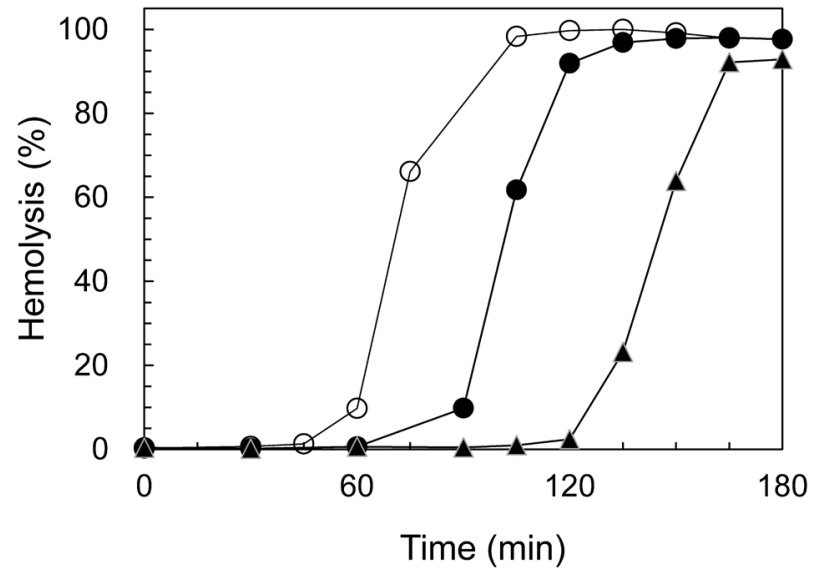

Fig. 2. Hemolysis of Erythrocytes by Treatment with AAPH and Inhibition by Ascorbic Acid

Open circles $(O)$ represent the hemolysis of erythrocytes caused by AAPHinduced oxidative membrane damage. The final reaction mixture comprised of $10 \%$ erythrocytes and $50 \mathrm{mM}$ AAPH. Hemolysis was suppressed by ascorbic acid $80 \mu \mathrm{M}, \boldsymbol{\Delta}: 240 \mu \mathrm{M})$. The reaction temperature was $37^{\circ} \mathrm{C}$. The extent of hemolysis (\%) was obtained using the ratio of the absorbance at $523 \mathrm{~nm}$ of the supernatant aliquot sample to that of the completely hemolyzed sample (water-treated). A typical result in multiple experiments is shown.

change in the absorbance with time is informative about both hemolysis and reaction of released $\mathrm{HbO}_{2}$ with AAPH (Fig. 1B). Instead, measuring the absorbance at 523 or $591 \mathrm{~nm}$ gives us information that only reflects the hemolysis induced by AAPH. Using the absorbance of the isosbestic point at $523 \mathrm{~nm}$ is preferable to $591 \mathrm{~nm}$ because intensity at $523 \mathrm{~nm}$ is larger than that at $591 \mathrm{~nm}$.

A typical example of the time course of hemolysis induced by AAPH is shown in Fig. 2. Hemolysis began to increase at around $60 \mathrm{~min}$, greatly increased at around $75-90 \mathrm{~min}$, and reached a plateau at around $120 \mathrm{~min}$. Addition of ascorbic acid delayed the beginning of hemolysis dose-dependently. Some other hydrophilic antioxidants, such as uric acid and $\alpha$-lipoic acid, also induced a similar delay in hemolysis, although the effective concentration range differed amongst each other (data not shown).

(-)-Epicatechin and edaravone (3-methyl-1-phenyl-2-pyrazolin-5-one; Radicut ${ }^{\circledR}$ ), which are also hydrophilic antioxidants, showed stronger suppression against AAPH-induced hemolysis than ascorbic acid did (Fig. 3). For the sample of $80 \mu \mathrm{M}$ (-)-epicatechin, the absorbance at $523 \mathrm{~nm}$ began to increase at $165 \mathrm{~min}$ due to hemolysis, whereas no increase was observed for the sample of $80 \mu \mathrm{M}$ edaravone. The suppression effect against AAPH-induced hemolysis was in the order edaravone $>(-)$-epicatechin $>$ ascorbic acid. (-)-Epicatechin showed the absorbance at $523 \mathrm{~nm}$ at early time, but the absorbance decreased after the maximum (at $30 \mathrm{~min}$ for $80 \mu \mathrm{M}$ and $75 \mathrm{~min}$ for $240 \mu \mathrm{M}$ ). These absorption at $523 \mathrm{~nm}$ observed at early time is not due to hemolysis because it decreased in time and the absorption spectrum was different from that observed by the hemolysis with AAPH (data not shown).

In order to assess the antioxidant activity of lipophilic compounds using the method of hemolysis of erythrocytes, we searched for appropriate agents that disperse lipophilic compounds to incorporate into the erythrocyte membranes. Dimethyl sulfoxide (DMSO), methylcellulose, and Tween 20 were examined, but all were found to be unsuitable. DMSO has antioxidant activity by itself, and methylcellulose and 
A)

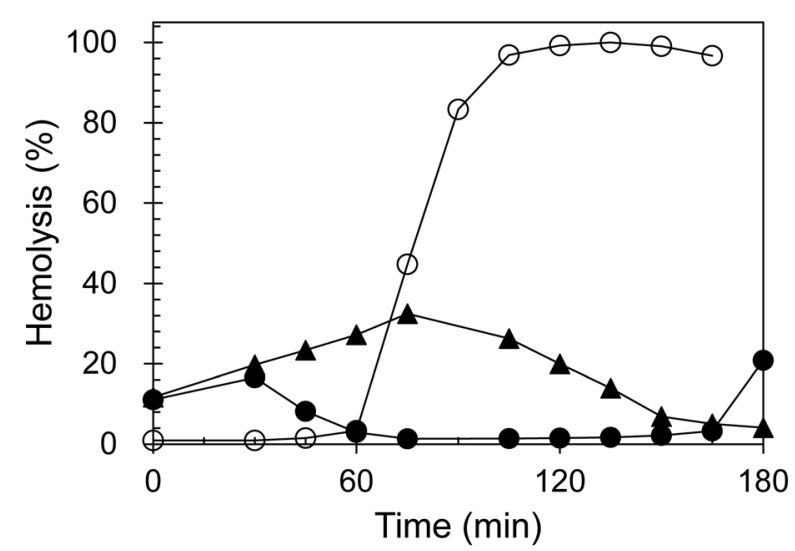

B)

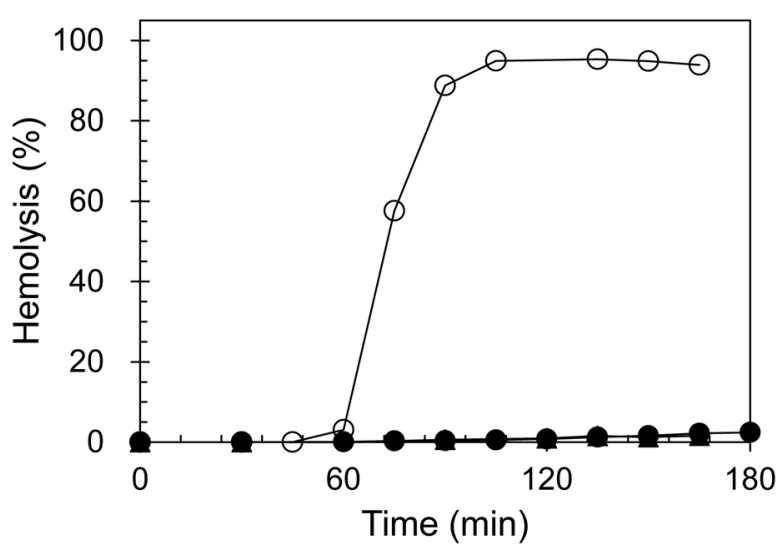

Fig. 3. Effects of (-)-Epicatechin and Edaravone on the Time Course of AAPH-Induced Hemolysis

Hemolysis was induced by $50 \mathrm{mM} \mathrm{AAPH}$ at $37^{\circ} \mathrm{C}$. A) Open circles (O) represent the hemolysis caused by AAPH-induced oxidative membrane damage in the absence of $(-)$-epicatechin. Closed circles $(-)$ represent the hemolysis induced by AAPH in the presence of $80 \mu \mathrm{M}(-)$-epicatechin. Closed triangles $(\boldsymbol{\Delta})$ represent the hemolysis induced by AAPH in the presence of $240 \mu \mathrm{M}(-)$-epicatechin. B) Open circles $(\bigcirc)$ represent the hemolysis caused by AAPH-induced oxidative membrane damage in the absence of edaravone. Closed circles $(-)$ represent the hemolysis induced by AAPH in the presence of $80 \mu \mathrm{M}$ edaravone. Closed triangles $(\mathbf{\Delta})$ represent the hemolysis induced by AAPH in the presence of $240 \mu \mathrm{M}$ edaravone. The extent of hemolysis (\%) was obtained using the ratio of the absorbance at $523 \mathrm{~nm}$ of the supernatant aliquot sample to that of the completely hemolyzed sample (watertreated). A typical result in multiple experiments is shown.

Tween 20 have strong detergent activity that induced hemolysis even before treatment with AAPH (data not shown). Next, we tried liposomes prepared with soybean phospholipids (asolectin) or 1,2-dimiristoyl-sn-glycero-3-phosphocholine (DMPC). Asolectin liposomes (as low as $0.75 \mathrm{mM}$ in concentration) accelerated the hemolysis induced by AAPH (data not shown). In contrast, DMPC liposomes (up to $0.74 \mathrm{mM}$ ) did not accelerate the AAPH-induced hemolysis. Figure 4 shows the effect of $0.34 \mathrm{mM}$ DMPC liposomes on the time course of hemolysis induced by $50 \mathrm{mM}$ AAPH.

Based on the above observations, we examined the antioxidant effect of a typical lipophilic antioxidant, $\alpha$-tocopherol $(\alpha$-Toc), on hemolysis using DMPC liposomes as a dispersing agent. DMPC liposomes at $0.34 \mathrm{mM}$ were used as a dispersing agent for various concentrations of $\alpha$-Toc in an $8.3 \%$ erythrocyte suspension. As shown in Fig. 5, the hemolysis induced by AAPH was inhibited by pretreatment of erythrocytes with

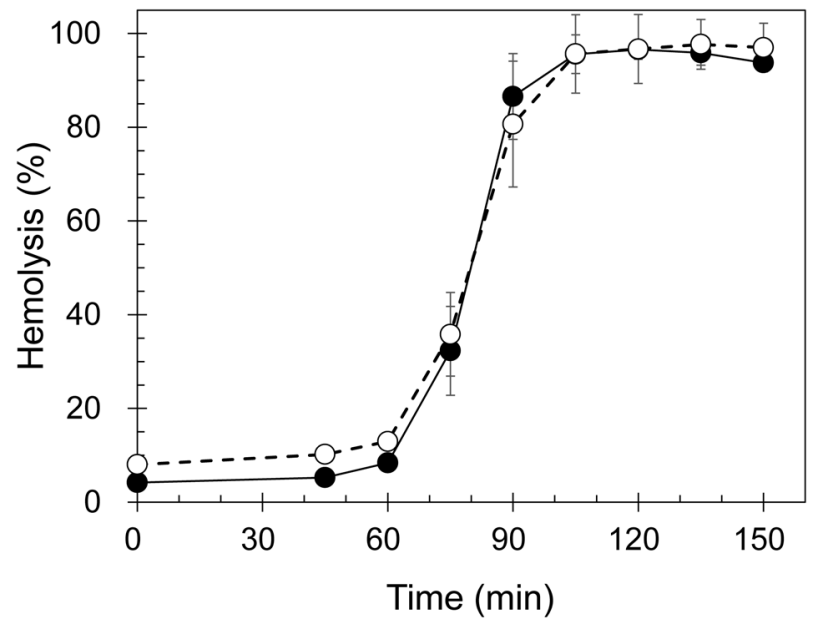

Fig. 4. Effect of DMPC Liposomes on the Time Course of AAPHInduced Hemolysis

Hemolysis was induced by $50 \mathrm{mM}$ AAPH at $37^{\circ} \mathrm{C}$ for the erythrocyte suspension with or without DMPC liposomes. Closed circles (-) represent the time-course of the AAPH-induced hemolysis of erythrocytes without DMPC liposome treatment, and open circles $(O)$ represent the time-course of the AAPH-induced hemolysis of erythrocytes treated with $0.34 \mathrm{mM}$ DMPC liposomes. The extent of hemolysis (\%) was obtained using the ratio of the absorbance at $523 \mathrm{~nm}$ of the supernatant aliquot sample to that of the completely hemolyzed sample (water-treated). Each point represents an average of three experiments and the bars represent standard deviation.

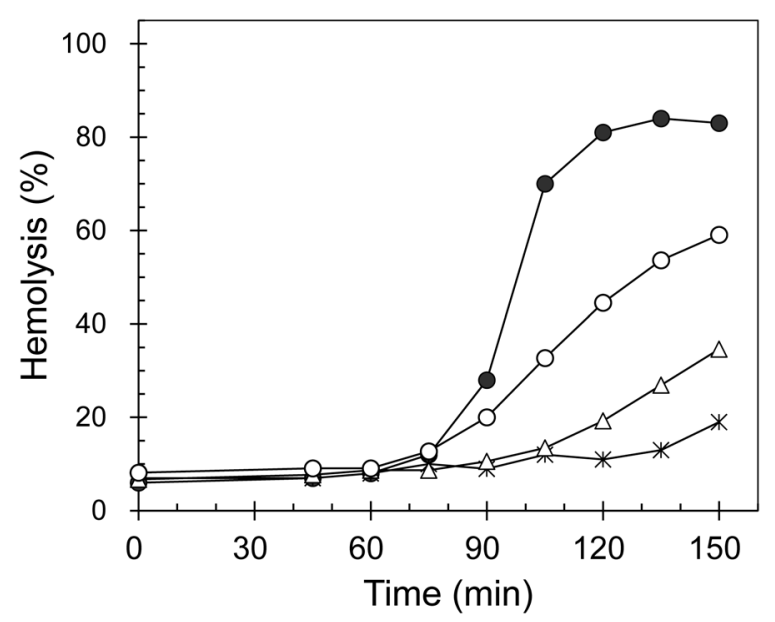

Fig. 5. Effect of $\alpha$-Toc on the Time Course of AAPH-induced Hemolysis

Hemolysis was induced by $50 \mathrm{mM} \mathrm{AAPH}$ at $37^{\circ} \mathrm{C}$. The erythrocyte suspension was treated with different concentrations of $\alpha$-Toc dispersed in $0.34 \mathrm{mM}$ DMPC liposomes. Closed circles (-) represent the time course of erythrocyte hemolysis without treatment with $\alpha$-Toc containing DMPC liposomes. The erythrocyte suspension was treated with DMPC liposomes containing $18 \mu \mathrm{M} \alpha$-Toc $(\bigcirc), 46 \mu \mathrm{M}$ $\alpha$-Toc $(\triangle)$, and $182 \mu \mathrm{M} \alpha$-Toc $(*)$. The extent of hemolysis (\%) was obtained using the ratio of the absorbance at $523 \mathrm{~nm}$ of the supernatant aliquot sample to that of the completely hemolyzed sample (water-treated). A typical result in multiple experiments is shown.

$\alpha$-Toc dispersed in DMPC liposomes. This effect was dependent on the concentration of $\alpha$-Toc.

\section{Discussion}

Hemolysis of erythrocytes is often measured by the optical absorbance of released $\mathrm{HbO}_{2}$. The wavelengths used for measuring the extent of hemolysis are around $400 \mathrm{~nm}$ (Soret-band) and 500-600 nm (Q-band). The absorption band at about $540 \mathrm{~nm}$ has often been used, ${ }^{34)}$ and use of this wavelength is reasonable because the band is relatively broad and displays sufficient intensity.

Thermal decomposition of AAPH firstly produces peroxyl 
radical $\left(\mathrm{ROO}^{\circ}\right)$ and further reaction produces alkoxyl radical $\left(\mathrm{RO}^{\circ}\right)$, which is suggested as the major species for the oxidation reaction of substrates. ${ }^{13)}$ For AAPH-induced oxidative hemolysis experiments, most of the published studies to date also used absorbance at about $540 \mathrm{~nm}$ to analyze the extent of hemolysis. ${ }^{8,10,11,14-30)}$ The absorption spectrum of erythrocytes that we observed after water-induced hemolysis had peaks at 541 and $576 \mathrm{~nm}$ (Fig. 1A). These two absorption maxima are derived from oxygenated hemoglobin $\left(\mathrm{HbO}_{2}\right)$, as reported previously. ${ }^{31-33)}$ As shown in Fig. $1 \mathrm{~B}, \mathrm{HbO}_{2}$ with a $\mathrm{Fe}^{2+}$ heme in the hemoglobin should be oxidized by AAPH, producing methemoglobin (metHb) with $\mathrm{Fe}^{3+}$ heme (an oxidized hemoglobin) after release from erythrocytes by hemolysis. ${ }^{35)}$ An absorption band at $630 \mathrm{~nm}$ was reported for metHb. ${ }^{31-33)}$ Since the absorbance at $540 \mathrm{~nm}$ of metHb is lower than that of $\mathrm{HbO}_{2}$ (Fig. 1A) and the formation of metHb is not very fast, we should not use the wavelength of about $540 \mathrm{~nm}$ to monitor the extent of hemolysis caused by AAPH. As shown in Fig. 1A, clear isosbestic points were observed at 523 and $591 \mathrm{~nm}$, indicating that absorbance at these wavelengths correctly reflects the amount of released hemoglobin regardless of the status of hemoglobin (oxidized or not oxidized). We used absorbance at $523 \mathrm{~nm}$ for the analysis of hemolysis in this study because the intensity at $523 \mathrm{~nm}$ is larger than that at $591 \mathrm{~nm}$.

We confirmed that AAPH induces oxidative hemolysis, as previously reported. ${ }^{8,10,11)}$ The hemolysis induced by AAPH was suppressed by ascorbic acid and uric acid dose-dependently, as reported by Niki et al. ${ }^{8)}$ It is reasonable to explain the suppression as follows: peroxyl radicals generated from AAPH were scavenged by ascorbic acid or uric acid in the aqueous phase until the antioxidant was used up. (-)-Epicatechin also showed dose-dependent antioxidant activity, as reported by Grzesik et al. ${ }^{36)}$ The activity was stronger than ascorbic acid. Edaravone also showed suppression of hemolysis induced by AAPH; this compound showed a very strong effect even at $80 \mu \mathrm{M}$ probably because it is a good scavenger of peroxyl radicals. ${ }^{37}$ )

There are many methods for measuring antioxidant activities of water-soluble compounds. However, only a relatively small number of methods have been reported to monitor antioxidant activity of lipophilic compounds. ${ }^{38-40)}$ Therefore, we attempted to identify a condition for applying the method of AAPH-induced oxidative hemolysis for assessing antioxidant activity of lipophilic compounds. Initially, several agents were examined to find appropriate agents for dispersing lipophilic compounds. Among them, DMPC liposome was found to be suitable for dispersing lipophilic compounds and incorporating them into erythrocytes membranes. DMPC liposome has been used previously by Niki et al., ${ }^{8)}$ but they observed enhancement of AAPH-induced hemolysis by $1.84 \mathrm{mM}$ DMPC liposome. In the present study, we also observed enhancement of AAPH-induced hemolysis by DMPC liposomes at similar concentrations, but lowering the concentration of DMPC liposomes improved the results; i.e., there was no effect on hemolysis. We found that concentrations lower than $0.74 \mathrm{mM}$ did not affect AAPH-induced hemolysis (Fig. 4).

This result was adopted to assess the method of oxidative hemolysis for testing the antioxidant effect of lipophilic compounds, using $\alpha$-Toc as a typical lipophilic antioxidant. AAPH-induced hemolysis was suppressed by the $\alpha$-Toc that was incorporated into the erythrocyte membranes by DMPC liposomes. $\alpha$-Toc as low as $18 \mu \mathrm{M}$ was effective for suppressing the AAPH-induced hemolysis. If we assume that all $0.34 \mathrm{mM}$ DMPC molecules and $18 \mu \mathrm{M} \alpha$-Toc molecules were incorporated evenly in the erythrocyte membranes of $8.3 \%$ erythrocyte suspension, then one erythrocyte is calculated to have about $5 \times 10^{6}$ DMPC molecules and about $3 \times 10^{4} \alpha$-Toc molecules. The inhibitory effect of $\alpha$-Toc against AAPHinduced hemolysis was reported by Miki et al. by comparing normal erythrocytes and $\alpha$-Toc deficient erythrocytes. ${ }^{11)}$ In the present study, we also found that exogenously-applied $\alpha$-Toc, by the use of DMPC liposomes, suppressed the AAPHinduced oxidative hemolysis.

In conclusion, we showed in the present study that the method of AAPH-induced oxidative hemolysis for assessing antioxidant and membrane-protection activities of lipophilic compounds may be improved by using: (1) the absorbance at $523 \mathrm{~nm}$ for monitoring AAPH-induced hemolysis, and (2) $0.34 \mathrm{mM}$ DMPC liposomes for dispersing the lipophilic compounds to apply to erythrocyte suspensions.

\section{Experimental}

Chemicals DMPC, asolectin, and $\alpha$-Toc were obtained from Sigma-Aldrich Co., Ltd., U.S.A. Edaravone (3-methyl-1-phenyl2-pyrazolin-5-one; Radicut ${ }^{\circledR}$ ) was a gift from Mitsubishi Pharma Co. (Tokyo, Japan). All other reagents were obtained from Wako Pure Chemical Corporation, Japan. All reagents were of the highest grade available and used without further purification.

Preparation of $\mathbf{2 0 \%}$ Erythrocyte Suspension Fresh horse blood was obtained from Nippon Bio-test Laboratories Inc. (Saitama, Japan) and diluted to a $20 \%$ suspension with $10 \mathrm{mM}$ phosphate buffered saline ( $\mathrm{pH}$ 7.4) (PBS). The suspension was centrifuged at $2000 \times \boldsymbol{g}$ for $5 \mathrm{~min}$ at room temperature, and erythrocytes were separated from the plasma and buffy coat. The pellet was resuspended with PBS to the original volume. This washing process was repeated three times and $20 \%$ erythrocyte suspension was prepared.

Measurement of Effect of Hydrophilic Antioxidants on AAPH-Induced Hemolysis of Erythrocytes The 20\% erythrocyte suspension with or without hydrophilic antioxidants and a solution of $100 \mathrm{mM}$ AAPH in $10 \mathrm{mM}$ PBS were pre-incubated separately at $37^{\circ} \mathrm{C}$ for $5 \mathrm{~min}$ in a water bath. The erythrocyte suspension $(5 \mathrm{~mL})$ was mixed with the same volume of AAPH solution and incubated in a water bath at $37^{\circ} \mathrm{C}$ in room light. The final concentrations were $10 \%$ erythrocytes and $50 \mathrm{mM}$ AAPH. An aliquot $(0.70 \mathrm{~mL})$ was taken out periodically and centrifuged at $5000 \times \boldsymbol{g}$ for $2 \mathrm{~min}$. Certain volumes of the supernatant were taken out and diluted with water to examine the extent of hemolysis by measuring the absorbance at $523 \mathrm{~nm}$ with a UV-visible spectrophotometer (Shimadzu UV-2550, Japan). Complete hemolysis was performed by diluting the erythrocyte suspension 10 -fold with water. The extent of hemolysis (\%) was calculated as the ratio of the absorbance at $523 \mathrm{~nm}$ of the sample to that of the complete hemolysis sample. For the experiments investigating the effect of hydrophilic antioxidants, the antioxidants were firstly mixed with the erythrocyte and then the AAPH solution was added.

Measurement of Effect of $\alpha$-Toc on AAPH-Induced Hemolysis of Erythrocytes For the experiments investigating the effect of hydrophobic $\alpha$-Toc, several reagents for suspend- 
ing $\alpha$-Toc were initially trialed and DMPC was found to be appropriate. Therefore, $\alpha$-Toc was suspended in DMPC liposomes, which were then applied to the erythrocyte suspension before mixing with the AAPH solution. Usually, a certain concentration of $\alpha$-Toc in chloroform was mixed with a chloroform solution of DMPC in a flask and then the chloroform was evaporated using a rotary evaporator. PBS was added to the flask and the mixture was sonicated for several minutes in a nitrogen atmosphere to make DMPC liposomes containing $\alpha$-Toc. The erythrocyte suspension $(20 \%, 2.5 \mathrm{~mL})$ was mixed with $0.5 \mathrm{~mL}$ of DMPC liposomes containing certain amounts of $\alpha$-Toc, or without $\alpha$-Toc, and incubated for $15 \mathrm{~min}$ at $37^{\circ} \mathrm{C}$, and then centrifuged at $2000 \times \boldsymbol{g}$ for $5 \mathrm{~min}$. The supernatant was removed and the pellet was resuspended in $10 \mathrm{mM}$ PBS up to the original volume $(3 \mathrm{~mL})$. The suspension $(3 \mathrm{~mL})$ was mixed with the same volume of $100 \mathrm{mM}$ AAPH solution and incubated in a water bath at $37^{\circ} \mathrm{C}$. The final concentrations were $8.3 \%$ erythrocytes, $50 \mathrm{mM}$ AAPH, and DMPC. An aliquot $(0.50 \mathrm{~mL})$ was taken out periodically to measure the extent of hemolysis with the UV-visible spectrophotometer (Shimadzu UV-2550, Japan).

Acknowledgments This work was supported in part by JSPS KAKENHI Grant Number 25450178 to KA.

Conflict of Interest The authors declare no conflict of interest.

\section{References}

1) Halliwell B., Gutteridge J. M. C., "Free Radicals in Biology and Medicine," 5th ed., Oxford University Press, U.K., 2015.

2) Janzen E. G., "Bioradicals Detected by ESR Spectroscopy," ed. by Ohya-Nishiguchi, H., Packer, L., Birkhauser Verlag, Basel, 1995, pp. 113-142.

3) Noda Y., Anzai K., Mori A., Kohno M., Shinmei M., Packer L., Biochem. Mol. Biol. Int., 42, 35-40 (1997).

4) Prior R. L., Wu X., Schaich K., J. Agric. Food Chem., 53, 4290 4302 (2005).

5) Cao G., Prior R. L., Methods Enzymol., 299, 50-62 (1999).

6) Re R., Pellegrini N., Proteggente A., Pannala A., Yang M., RiceEvans C., Free Radic. Biol. Med., 26, 1231-1237 (1999).

7) Benzie I. F. F., Strain J. J., Anal. Biochem., 15, 70-76 (1996).

8) Niki E., Komuro E., Takahashi M., Urano S., Ito E., Terao K., J. Biol. Chem., 263, 19809-19814 (1988).

9) Yamamoto Y., Niki E., Eguchi J., Kamiya Y., Shimasaki H., Biochim. Biophys. Acta, 819, 29-36 (1985).

10) Yamamoto Y., Niki E., Eguchi J., Kamiya Y., Miki M., Tamai H., Mino M., J. Nutr. Sci. Vitaminol., 32, 475-479 (1986).

11) Miki M., Tamai H., Mino M., Yamamoto Y., Niki E., Arch. Biochem. Biophys., 258, 373-380 (1987).

12) Niki E., Kawakami A., Yamamoto Y., Kamiya Y., Bull. Chem. Soc. Jpn., 58, 1971-1975 (1985).

13) Werber J., Wang Y. J., Milligan M., Li X., Ji J. A., J. Pharm. Sci., 100, 3307-3315 (2011).

14) Pieroni L. G., de Rezende F. M., Ximenes V. F., Dokkedal A. L., Molecules, 16, 9439-9450 (2011).

15) Ilavenil S., Kaleeswaran B., Sumitha P., Tamilvendan D., Ravikumar S., Saudi J. Biol. Sci., 18, 181-187 (2011).
16) Hasnat M. A., Pervin M., Lim B. O., Molecules, 18, 6663-6678 (2013).

17) Hou X., Zhang J., Ahmad H., Zhang H., Xu Z., Wang T., PLOS ONE, 9, e108314 (2014).

18) Casagrande J. C., Macorini L. F. B., Antunes K. A., dos Santos U. P., Campos J. F., Dias-Júnior N. M., Sangalli A., Lima Cardoso C. A., do Carmo Vieira M., Rabelo L. A., Paredes-Gamero E. J., dos Santos E. L., de Picoli Souza K., PLOS ONE, 9, e112748 (2014).

19) Bonfanti G., De Bona K. S., de Lucca L., Jantsch L., Pigatto A. S., Boligon A. A., Athayde M. L., Moretto M. B., Gonçalves T. L., Redox Rep., 19, 206-213 (2014).

20) Qasim N., Mahmood R., PLOS ONE, 10, e0141975 (2015).

21) Santos U. P., Campos J. F., Torquato H. F. V., Paredes-Gamero E. J., Carollo C. A., Estevinho L. M., Souza K. P., dos Santos E. L., PLOS ONE, 11, e0167531 (2016).

22) Lopes R. H. O., Macorini L. F. B., Antunes K. Á., Espindola P. P. T., Alfredo T. M., da Rocha P. S., Pereira Z. V., dos Santos E. L., Souza K. P., Oxid. Med. Cell. Longev., 2016, 9681425 (2016).

23) Campos J. F., de Castro D. T. H., Damião M. J., Torquato H. F. V., Paredes-Gamero E. J., Carollo C. A., Estevinho L. M., Souza K. P., dos Santos E. L., Oxid. Med. Cell. Longev., 2016, 8405957 (2016).

24) Salazar-López N. J., González-Aguilar G. A., Loarca-Piña G., Cinco-Moroyoqui F. J., Rouzaud-Sández O., Domínguez-Avila J. A., Robles-Sánchez M., Oxid. Med. Cell. Longev., 2017, 8219023 (2017).

25) Li M., Chen Y., Zhang P., Zhang L., Zhou R., Xu Y., Ding H., Wang Q., Wang Z., Chem. Pharm. Bull., 66, 535-540 (2018).

26) dos Santos J. M., Alfredo T. M., Antunes K. A., da Cunha J. S. M., Costa E. M. A., Lima E. S., Silva D. B., Carollo C. A., Schmitz W. O., Boleti A. P. A., dos Santos E. L., Souza K. P., Oxid. Med. Cell. Longev., 2018, 2935051 (2018).

27) Xin X., Zhang M., Li X., Lai F., Zhao G., Microb. Cell Fact., 17, 130 (2018).

28) Zhang J., Han H., Shen M., Zhang L., Wang T., Animals, 9, 953 (2019).

29) Firpo G., Ramírez M. L., Faillace M. S., de Brito M. R. M., Silva A. P. S. C. L., Costa J. P., Rodríguez M. C., Argüello G. A., Szakonyi Z., Fülöp F., Peláez W. J., Antioxidants, 8, 197 (2019).

30) Du R., Liu K., Zhao S., Chen F., ACS Omega, 5, 12751-12759 (2020).

31) van Assendelft O. W., "Spectrophotometry of haemoglobin derivatives," Charles C. Thomas, Springfield, IL, 1970.

32) Zijlstra W. G., Buursma A., Comp. Biochem. Physiol. B, 118, $743-$ 749 (1997).

33) Zijlstra W. G., Buursma A., van Assendelft O. W., "Visible and Near Infrared Absorption Spectra of Human and Animal Haemoglobin," VSP, Utrecht, 2000.

34) Mino M., Kitagawa M., Nakagawa S., J. Nutr. Sci. Vitaminol., 27, 199-207 (1981).

35) Celedón G., Rodriguez I., España J., Escobar J., Lissi E., Free Radic. Res., 34, 17-31 (2001).

36) Grzesik M., Naparło K., Bartosz G., Sadowska-Bartosz I., Food Chem., 241, 480-492 (2018)

37) Yamamoto Y., Kuwahara T., Watanabe K., Watanabe K., Redox Rep., 2, 333-338 (1996).

38) Takahashi Y., Ichimori K., Okano M., Goto H., J. Clin. Biochem. Nutr., 56, 105-110 (2015).

39) Mukai K., J. Nutr. Sci. Vitaminol., 65, 285-302 (2019).

40) Cano A., Acosta M., Arnao M. B., Redox Rep., 5, 365-370 (2000). 